\title{
Considerations on localization of macroscopic bodies
}

\author{
B. Carazza \\ Dipartimento di Fisica dell' Università, viale delle Scienze, I43100 Parma, \\ Italy \\ INFN Sezione di Cagliari, Italy
}

\begin{abstract}
Position holds a very special role in understanding the classical behaviour of macroscopic bodies on the basis of quantum principles. This lead us to examine the localised states of a large condensed object in the context of a realistic model. Following the argument that an isolated macroscopic body is usually described by a linear superposition of low-lying energy eigenstates, it has been found that localised states of this type correspond to a nearly minimum-uncertainty state for the center of mass. An indication is also given of the dependence of the center of mass position spread on the number of constituent particles. This paper is not offered as an answer to the intriguing question of the preferred role played by the position basis, but will hopefully provide some contribution to the quantum modelling of multiparticle systems.
\end{abstract}

Key words: Macroscopic states, spatial macrosuperpositions, preferred basis, approximate position eigenstates. 


\section{INTRODUCTION}

Classical physics has explained a variety of phenomena, ranging from sound to heat, in terms of systems of moving particles. These achievements are firmly based on the primary notions of position and trajectory first deduced from the observation of common everyday objects. It was possible to do this because macroscopic bodies appear to be fairly accurately localised, which means that position undoubtedly plays a privileged role in our knowledge of the external world. However, if the problem is approached in terms of quantum physics, disregarding Bohr's point of view and applying it to all objects (not only microscopic ones), a number of difficulties are encountered.

We could assume, when looking at the moon, that we see it in a certain position because it is described by a wave packet that is well localised precisely there, and not because our glance caused the moon's wave packet to collapse into that state. Generally speaking, it may be wondered why spatial macrosuperpositions have not been encountered, even though they are theoretically possible, whereas macroscopic objects do tend to be found in (approximate) eigenstates of position.

All this concerns the general debate about the classical limits of quantum formalism. Recent work on the subject has been successful thanks to the use, for instance, of the functional representation of Hilbert space in terms of coherent states [1], the Wigner function [2] and, above all, such concepts

as decoherence [3]. Many researchers believe that decoherence analysis also adequately explains why position is so important.

The best result we can hope for is to demonstrate that, within the macroscopic limit, a body is usually described by a statistical mixture, each element of the ensemble being associated with a pure state corresponding, for all practical purposes, to a well defined position of its center of mass. Efforts in this direction have reached their objective not only through application of the decoherence mechanism but also by using the model of spontaneous dynamical reduction proposed by Ghirardi, Rimini and Weber [4], later refined as the Continuous Spontaneous Localisation model (CSL) [5]. The CSL model requires selection of the so-called preferred basis- i.e. the eigenmanifold in which reduction occurs - and the authors attribute this privileged role to position.

In conclusion, daily experience, common sense and theoretical studies 
all attribute preferred status to the position of a macroscopic object. The present paper is intended as a supplement to the debate about this intriguing role, a debate which also extends into the realms of philosophy. Our intention is not to explain why large objects tend to be found in approximate eigenstates of position but, since well localised states of macroscopic bodies evidently do exist, the more modest purpose is to examine their quantum properties and discover whether they normally possess certain characteristics. This will be applied to the case of an isolated macroscopic object, going beyond such familiar models as coupled harmonic oscillators [6].

\section{THE NATURAL STATES OF A MACROSCOPIC BODY}

Let us consider a large isolated object. We can attempt to answer the following question: in what quantum mechanical state can an isolated macroscopic body ordinarily be found? Although interaction of the system with its surroundings is so small that it can be considered isolated, it must be admitted - with Landau and others [7]- that a macroscopic body can, in fact, never be in a strictly stationary state, due to the extraordinary density of its energy levels and to the action of surrounding objects, even if this tends towards zero. In other words, macroscopic objects are never at absolute zero temperature. Yet their temperatures must be much lower the typical atomic binding energies which hold them together, in order to ensure the permanence and stability usually associated with a "body".

If macroscopic objects are in contact with their environment, they inevitably undergo the apparent GRW localisation caused by decoherence. This apparent collapse of the wave function may be caused, for example, by random scattering [8]. However, the effects of the external environment

on dynamics are not considered here. Since we wish to identify the type of pure state in which the macroscopic object is generally found, it will only be assumed, for the reasons stated above, that it is a linear superposition of low-lying eigenstates of total energy in a reference frame where average total momentum is zero. This is a means of taking into account the external environment at zeroth order, while the specification about the reference frame is necessary in that the total energy is not a Galilean invariant. Such linear superpositions will be called "natural states".

This manner of proceeding allows the mixing of internal energy levels 
with the kinetic energy of the center of mass. In the present case what is considered as a difficulty within the nuclear shell model [9] is actually welcome. Let us now look at the properties of localised macroscopic systems with average energies slightly higher than the ground state. This will be done with reference to a realistic model.

\section{THE MODEL OF A MACROSCOPIC BODY}

Let us now consider a system of $N$ spinless identical bosons with mass $\mu$ interacting in pairs through a two-body potential of the Van der Waals type - i.e., with a long-range attractive part and a short-range repulsive one. This corresponds to a realistic picture for an aggregation of noble gas atoms, as long as all of them are in their ground state and can thus be considered constituent elements. It is impossible to solve the eigenvalue problem for such a system in order to investigate a generic superposition of low- lying energy states. However, we may proceed by applying the variational method to determine the parameters of a trial wave function, requiring that the average value of the Hamiltonian should be minimum. The possibility that this trial wave function is the ground state wave vector or corresponds exactly to a stationary state is minimal. It will basically be a linear superpositions of stationary states, the lower ones of which will presumably dominate.

This can now be used to calculate the spread of the center of mass position. The trial state vector considered is the normalised and completely symmetrised state constructed from the product:

$$
\prod_{i=1}^{N} \varphi_{i}\left(\boldsymbol{r}_{i}\right)
$$

where each function $\varphi_{i}\left(\boldsymbol{r}_{i}\right)$ is centred on the $i^{\text {th }}$ of the $N$ contiguous lattice points $\boldsymbol{x}_{i}$ of a portion of regular lattice surrounded by a spherical surface whose origin coincides with the origin of the coordinates. Apart from this, all of the functions $\varphi_{i}\left(\boldsymbol{r}_{i}\right)$ have the same exponential-type form:

$$
\varphi_{i}(\boldsymbol{r})=\left\{\begin{array}{lc}
D e^{-\lambda / 2\left|\boldsymbol{x}_{i}-\boldsymbol{r}\right|} & \text { for }\left|\boldsymbol{x}_{i}-\boldsymbol{r}\right| \leq a \\
0 & \text { otherwise }
\end{array}\right.
$$

where $D$ is the normalisation factor and $a$ is a quantity lower than or equal to half the nearest lattice spacing. 
Given this condition, two functions of the set referring to different sites on the lattice do not overlap and are therefore orthogonal. The usual second quantisation formalism can therefore be used without modification to deal with a system of identical bosons. The average value of the Hamiltonian is expressed with one- and two-body terms as follows:

$$
\begin{gathered}
<H>=\sum_{i} \frac{1}{2 \mu} \int \varphi_{i}^{*}(\boldsymbol{r}) \widehat{p^{2}} \varphi_{i}(\boldsymbol{r}) d^{3} r \\
+\frac{1}{2} \sum_{i} \sum_{j} \iint \varphi_{i}^{*}(\boldsymbol{r}) \varphi_{i}(\boldsymbol{r}) v\left(\left|\boldsymbol{r}-\boldsymbol{r}^{\prime}\right|\right) \varphi_{j}^{*}\left(\boldsymbol{r}^{\prime}\right) \varphi_{j}\left(\boldsymbol{r}^{\prime}\right) d^{3} r d^{3} r^{\prime}
\end{gathered}
$$

where $\widehat{p^{2}}=\widehat{\boldsymbol{p}} \cdot \widehat{\boldsymbol{p}}$. Here, $\widehat{\boldsymbol{p}}$ is the one particle momentum operator and $v\left(\left|\boldsymbol{r}-\boldsymbol{r}^{\prime}\right|\right)$ is the two-body potential.

The expression for average potential energy can be re-written as

$$
\frac{1}{2} \sum_{i} \sum_{n^{(i)}} c_{n^{(i)}} \iint \varphi_{i}^{*}(\boldsymbol{r}) \varphi_{i}(\boldsymbol{r}) v\left(\left|\boldsymbol{r}-\boldsymbol{r}^{\prime}\right|\right) \varphi_{n^{(i)}}^{*}\left(\boldsymbol{r}^{\prime}\right) \varphi_{n^{(i)}}\left(\boldsymbol{r}^{\prime}\right) d^{3} r d^{3} r^{\prime}
$$

The second sum is on the neighbouring $n^{(i)}$ of each site $i$, and $c_{n^{(i)}}$ indicates the number of the $n^{t h}$ next nearest . Since the realistic potential to be used goes rapidly to zero, it can be further simplified with accuracy by truncating the sum on the neighbours at a specific $\bar{n}$ and ignoring the surface effects for large $N$.

The result for the average value of the Hamiltonian is then:

$$
\begin{gathered}
<H>=\frac{N}{2 \mu} \int \varphi_{l}^{*}(\boldsymbol{r}) \widehat{p^{2}} \varphi_{l}(\boldsymbol{r}) d^{3} r+ \\
\frac{N}{2} \sum_{n^{(l)}}^{\bar{n}} c_{n^{(l)}} \iint \varphi_{l}^{*}(\boldsymbol{r}) \varphi_{l}(\boldsymbol{r}) v\left(\left|\boldsymbol{r}-\boldsymbol{r}^{\prime}\right|\right) \varphi_{n^{(l)}}^{*}\left(\boldsymbol{r}^{\prime}\right) \varphi_{n^{(l)}}\left(\boldsymbol{r}^{\prime}\right) d^{3} r d^{3} r^{\prime}
\end{gathered}
$$

where the suffix $l$ indicates a generic lattice point. The one-body term (i.e. average kinetic energy) can be expressed analytically. A closed result for the double integral expressing the average potential energy is more difficult to obtain. However, this can be expressed by a single integration in the Fourier space, taking into account the isometric properties of the Fourier transform and using the convolution theorem for the quantity:

$$
U\left(\boldsymbol{r}^{\prime}\right)=\int v\left(\left|\boldsymbol{r}-\boldsymbol{r}^{\prime}\right|\right) \varphi_{n^{(l)}}^{*}\left(\boldsymbol{r}^{\prime}\right) \varphi_{n^{(l)}}\left(\boldsymbol{r}^{\prime}\right) d^{3} r^{\prime} \quad .
$$

Integration was then easily performed numerically. 
The $N$ bosons were then considered as Krypton atoms. Thus, the corresponding value was used for mass $\mu$. As for two-body interaction, the two Yukawa potential was used as follows:

$$
v(r)=-\epsilon b\left[e^{-m(r / \sigma-1)}-e^{-n(r / \sigma-1)}\right] /(r / \sigma)
$$

with $b=2.026, m=2.69$ and $n=14.70$. The above expression is well known in scientific literature and is widely used for the best possible reproduction of the Van der Waals potential in the case of noble gases [10]. In the case of Krypton, $\epsilon=170 \mathrm{~K}$ and $\sigma=3.6 \AA$. As can be seen in Eq. (5), the average energy is additive, as is to be expected. This energy depends on the type of lattice, its nearest spacing $d$ and parameters $\lambda$ and $a$ relative to the function $\varphi(\boldsymbol{r})$.

After some work on the computer, the minimum value for average energy was obtained in the case of a face centred cubic lattice with the nearest neighbour distance $d=3.953 \AA$ and when $\lambda=91.33 / \sigma$. Parameter $a$ was fixed at the beginning as equal to half of $d$. However, suitable values of $\lambda$ are so large and $\varphi(\boldsymbol{r})$ therefore decreases so rapidly (in accordance with the initial assumption of non-overlapping) that $a$ can safely be left to go on to infinity.

Once the probe function was characterised, it was easy to deduce both cohesive energy $U$ and bulk modulus $B$. The results obtained were: $U=-2690$ $\mathrm{cal} / \mathrm{mole}$ and $B=33.4 \mathrm{kbar}$. The experimental values for solid Krypton at $0 \mathrm{~K}$, which actually crystallises as a face centred cubic, are indicated [11] as $d=3.992 \AA, U=-2666 \mathrm{cal} / \mathrm{mole}$ and $B=34.3 \mathrm{kbar}$. Although it was not the aim here to make a solid state computation, comparison of the results with the experimental ones suggests that the probe state specified above represents a combination of low-lying states.

A function $\varphi(\boldsymbol{r})$ centred on the ith reticular point can, of course, be associated with more than one of the spinless bosons. To see how this can affect the average energy, the following quantity must be considered:

$$
W=\iint \varphi_{i}^{*}(\boldsymbol{r}) \varphi_{i}(\boldsymbol{r}) v\left(\left|\boldsymbol{r}-\boldsymbol{r}^{\prime}\right|\right) \varphi_{i}^{*}\left(\boldsymbol{r}^{\prime}\right) \varphi_{i}\left(\boldsymbol{r}^{\prime}\right) d^{3} r d^{3} r^{\prime} \quad .
$$

If $p$ particles occupy the same lattice site, the contribution to the average potential energy from their interaction is $p(p-1) W / 2$. In the case of a potential with a short-range repulsive part, this contribution is presumably positive. In the present case, with the indicated values of $\lambda$ and $d, W$ is 
not only positive, but also ten million times greater than the absolute value of the (negative) interaction energy per particle. It can be concluded that the lowest value for the average energy of the system is reached in the case considered of only one particle per site. This conclusion does not change in the case of bosons with non-zero spin.

\section{THE SPREAD OF THE CENTER OF MASS POSITION}

Using the probe wave function obtained, we can calculate the spread of the center of mass position. Components $R_{i}$ and $P_{i}$ of the center of mass position vector:

$$
\boldsymbol{R}=\sum \boldsymbol{r}_{i} / N
$$

and of the conjugate momentum:

$$
\boldsymbol{P}=\sum \boldsymbol{p}_{i}
$$

have an average value of zero. So the requirement on the mean value of the total momentum is satisfied. The average squares of $R_{i}$ are:

$$
<R_{i}^{2}>=\frac{4}{\lambda^{2} N} \quad(i=1,2,3) \quad .
$$

The mean square deviations:

$$
\chi_{i}=<R_{i}^{2}>-<R_{i}>^{2}=\frac{4}{\lambda^{2} N} \quad(i=1,2,3)
$$

therefore depend on $N$ as $1 / N$. The following is obtained for the mean-square deviations of the momentum components:

$$
\omega_{i}=<P_{i}^{2}>-<P_{i}>^{2}=\hbar^{2} \lambda^{2} N / 12 \quad(i=1,2,3) \quad .
$$

These are proportional to $N$ and it can be seen that the indeterminacy product is very close to the minimum value.

It can be observed that the mean-square deviations for the center of mass velocity components behave like $1 / N$. In other words, both of the collective (pseudo)conjugate variables represented by the center of mass coordinate and its velocity have vanishing small dispersion in the limit of large $N$. 
We have concentrated so far on the properties of the state vector obtained, which will be indicated as $\Phi\left(\boldsymbol{r}_{1}, \boldsymbol{r}_{2}, \cdots\right)$. However, since the origin and orientation of the coordinates are arbitrary, the states which can be obtained from it by translation and rotation must also be considered. The Hamiltonian is translationally and rotationally invariant, so these states have the same average energy. It is particularly interesting to investigate a linear superposition of them. For the sake of simplicity, we have considered only a superposition of translated states:

$$
\begin{array}{r}
\Psi\left(\boldsymbol{r}_{1}, \boldsymbol{r}_{2}, \cdots\right)=c_{0} \Phi\left(\boldsymbol{r}_{1}, \boldsymbol{r}_{2}, \cdots\right)+c_{1} \Phi\left(\boldsymbol{r}_{1}+\boldsymbol{a}_{1}, \boldsymbol{r}_{2}+\boldsymbol{a}_{1},+\cdots\right)+\cdots \\
\cdots+c_{n} \Phi\left(\boldsymbol{r}_{1}+\boldsymbol{a}_{n}+\boldsymbol{r}_{2}+\boldsymbol{a}_{n}, \cdots\right) .
\end{array}
$$

The above wave vector is still a completely symmetrised state of the boson system. Let us consider the very simple case in which all the $\Phi$ in the second member are centred so far apart from each other that none of them overlaps another. This is possible due to the way in which the functions $\varphi_{i}(\boldsymbol{r})$ appearing in the product (Eq. (1)) defining the trial vector were chosen. In these conditions, the cross terms do not contribute to average energy or to average total momentum. Furthermore, the scalar product of each pair of $\Phi$ centred at different points is zero and the normalisation condition requires:

$$
\sum_{i=0}^{n}\left|c_{i}\right|^{2}=1
$$

So the linear superposition considered has the same average energy as the starting state and the average value of the center of mass momentum is still zero. The $\Psi\left(\boldsymbol{r}_{1}, \boldsymbol{r}_{2}, \cdots\right)$ corresponds to the entire blob of solid Krypton being in a spatial superposition of localised states, which can be separated by any distance, however great. The resulting spread for the center of mass position is therefore unlimited, which unfortunately means that our energy considerations cannot be used to select only localised states.

It can be observed that, under the present conditions, the matrix element between two different $\Phi$ is zero not only in the case of energy and total momentum but also for any observable depending on the coordinates and momenta of the particles. This is the basic requisite of superselection rules for spatial macrosuperpositions of our localised states. However, it is better not to insist on this, since everything depends on having considered the initial functions $\varphi$ as equal to zero outside a finite spatial region. 


\section{THE SELF GRAVITATING PARTICLES}

The system examined here is unlikely to represent a generic macroscopic body, even though it may be thought that interaction between particles with both a short-range repulsive part and a long-range attractive one is an essential characteristic as long as atoms or molecules of the same type can be considered as elementary constituents of a large homogeneous object.

One way to generalise our model of a body is to consider the case in which the elementary constituents are identical fermions with spin $s$. A lattice site may then be occupied by up to $g=2 s+1$ particles with different spin states. But reference can be made to our previous considerations on this type of configuration: it was seen that the contribution to potential average energy is positive and very large when compared with the absolute value of (negative) average energy per particle in the case of one particle per site. Looking for a superposition of low-lying energy states, as long as we consider a spin independent two-body interaction of Van der Waals type, it does not matter whether the constituents of the body are bosons or fermions. The localised "natural states" being sought correspond to only one particle per site, whether a boson or a fermion, irrespective of the spin state the particle assumes there. In the present model, the spread of the center of mass position for a localised state with low average energy is therefore the same for bosons and fermions.

This independence from the type of constituent particles is presumably due to the type of potential used, with a deep minimum at finite distance. To see whether the situation changes for other two-body potentials, let us examine a system of identical self gravitating particles. The resulting model of a macroscopic body is not very realistic, but it may throw light on the point in question. The pair wise interaction is now:

$$
v\left(\left|\boldsymbol{r}-\boldsymbol{r}^{\prime}\right|\right)=-\frac{\kappa}{\left|\boldsymbol{r}-\boldsymbol{r}^{\prime}\right|} .
$$

Let us first consider the case of identical spinless bosons. When searching for a localised state of very low average energy, all particles should preferably be in the same spatial state, as has been seen in the case of the (relativistic) collapse of a boson star [12]. The following $N$-body wave function (which 
was useful in the case of boson stars) is used as the trial state vector:

$$
\Theta\left(\boldsymbol{r}_{1}, \boldsymbol{r}_{2}, \cdots\right)=D(\beta) \prod_{i=1}^{N} e^{-\beta r_{i}}
$$

where $D(\beta)$ is the normalisation factor. Corresponding average energy is given by:

$$
<E>=N \hbar^{2} \beta^{2} /(2 \mu)-5 \kappa N(N-1) \beta / 16
$$

where $\mu$ is the mass and $N$ the number of particles. The minimum value is reached when:

$$
\beta=5 \kappa \mu(N-1) /\left(16 \hbar^{2}\right) \quad .
$$

Now the average energy is no longer proportional to $N$, a result already known [14]. The following average values for the center of mass coordinates are obtained:

$$
\begin{gathered}
<R_{i}>=0 \quad(i=1,2,3) \\
<R_{i}^{2}>=g^{2} /\left(N(N-1)^{2}\right) \quad(i=1,2,3)
\end{gathered}
$$

where $g=16 \hbar^{2} /(5 \kappa \mu)$. The mean-square deviations of the center of mass position components depend on $N$ as $1 / N^{3}$. The mean-square deviations of the components of momentum are proportional to $N(N-1)^{2}$, while the indeterminacy product is equal to $\hbar / \sqrt{3}$.

If the self gravitating particles are fermions, an anti- symmetrised wave function must be used as a trial state vector. In this case, the calculations would be complicated and it is therefore preferable to resort to semi-classical approximation, finding the minimum of the Thomas-Fermi energy functional:

$$
\int \tau(\boldsymbol{r}) d^{3} r-\frac{\kappa}{2} \iint \frac{\rho(\boldsymbol{r}) \rho\left(\boldsymbol{r}^{\prime}\right)}{\left|\boldsymbol{r}-\boldsymbol{r}^{\prime}\right|} d^{3} r d^{3} r^{\prime}
$$

with

$$
\tau(\boldsymbol{r})=\frac{e \hbar^{2}}{q^{2 / 3} \mu} \rho^{5 / 3}(\boldsymbol{r})
$$

where $e \simeq 5$ is a numerical factor, $\rho(\boldsymbol{r})$ is the spatial distribution of the particles and $q$ is the occupation number. 
We assumed the following trial spatial distribution normalised to the number $N$ of fermions:

$$
\rho(\boldsymbol{r})=N \gamma^{3} e^{-\gamma r} /(8 \pi)
$$

where $\gamma$ is a parameter to be varied. When considering particles with $1 / 2$ spin $(q=2)$, it is found that the minimum of Eq. (22) (i.e. of average

energy) is reached when $\gamma=f \kappa \mu N^{1 / 3} / \hbar^{2}, f$ being a numerical factor of order unity. With the aid of the corresponding spatial particle distribution, the mean-square deviations for the center of mass position components can be calculated. The following is obtained:

$$
\chi_{i}=\frac{4 \hbar^{2}}{b^{2} \kappa^{2} \mu^{2} N^{5 / 3}} \quad(i=1,2,3) \quad .
$$

It can be seen that fermions and bosons behave differently as regard the spread of the centre of mass position. In both cases, however, the position spread decreases as $N$ increases.

\section{FINAL REMARKS}

A brief summary of the findings in the case of our model of macroscopic body is needed here. For the states identified using our trial wave function and our energy considerations, the average total momentum is zero and the center of mass position is well localised. A Galilean transformation [13 can also be applied to provide the body with a required average momentum (velocity). The state considered can also be slightly modified, for example by changing the value of $\lambda$ for some or all of the functions $\varphi(\boldsymbol{r})$ centred on the lattice points or by allowing some other points of the lattice to be occupied and some vacancies to appear. By applying a translation and a Galilean transformation, it is then possible to have various states of an isolated body which correspond to a well defined position and velocity of the center of mass.

These states correspond to a nearly minimum-uncertainty state for the center of mass, and the mean-square deviations of the center of mass position components decrease as $1 / N$ as the number $N$ of constituents increases. The above property does not seem to differ whether the constituent particles are bosons or fermions. Unfortunately, there are a large number of states which 
have the same low average energy and are not at all localised. These are given by the macrosuperpositions of the previous states centred in different places and with different orientations. Apart from this, since the body is isolated, the spread of the center of mass position increases in any case with the passing of time as $t^{2}$. To obtain a classical behaviour in the macroscopic limit, it is evidently necessary to resort to mechanisms such as decoherence caused by the external environment. We have obtained macroscopic states which are basically eigenstates of position ( although these constitute a set which has measure zero compared to all other delocalised states with the same energy ), starting with considerations regarding the inevitable interaction of the body with surrounding objects. This at least seems to indicate that we were moving in the right direction.

Since the criterion adopted was not sufficient to select only localised states, we asked ourselves what further requirement was needed to reach this objective. It may be conjectured that besides being a superposition of low-lying energy states, the macroscopic states must be a product of the wave functions of the individual constituents. However, it is not clear how this condition can be justified.

The case of a system of $N$ self gravitating particles was then investigated. For those localised trial states which satisfy our energy criterion and in the case of bosons, the mean-square deviations of the center of mass position components decrease as $1 / N^{3}$ and the indeterminacy product is close to the minimum value. In the case of fermions, the center of mass is still well localised but the mean- square deviations of its position components decrease as $1 / N^{5 / 3}$.

In conclusion we have not, of course, answered the big question about the special role which position seems to play in the macroscopic limit of quantum mechanics. However, there does seem to be some merit in demonstrating, within our solid noble gas model, the plausibility of well localised macroscopic bodies corresponding to a minimum uncertainty product for their center of mass.

Of course we cannot consider to be generally valid the result we obtained in this connection by a trial wavefunction which is not a very generic wavefunction of the desidered kind. It was selected mainly on grounds of computational convenience and having in mind the classical ground state. But since it would be very energetically expensive to have any inter-particle separation differing greatly from the classical arrangement, a " natural state 
" of the system considered cannot have any of its constituent particles in a flagrant macrosuperposition, except those that reflect the symmetries of the Hamiltonian, in our case global translation, rotation and reflection of all particles together. These considerations seems indicate that the localised state of the system cannot differ greatly from our trial wave function if its energy is to be close to minimal. So the conclusion that states with uncertainty product close to minimal are in a sense singled out by energy considerations may be generally correct.

It interesting to note at last that the study of the apparent collapse of the wave function caused by scattering [8] seems to indicate that macroscopic bodies tend to be characterised by a minimum packet.

\section{References}

[1] R.J. Klauder Commun. Math. Phys., 5, 177 (1964)

[2] E.P. Wigner, Phys. Rev., 40,479 (1932)

[3] W. H. Zurek, Phys. Rev. D 26, 1862 (1982); E. Joos, H. D. Zeh Zeits. Phys. B 59, 223 (1985); A. O. Caldeira, A. J. Legget Phys. Rev. A 31, 1059 (1985); W. H. Zurek, Physics Today 44 , 36 (1991), ibid,46, 81 (1993); W. H. Zurek,Prog. Theor. Phys. 89, 281 (1993); J. P. Paz, W.

H. Zurek Phys. Rev. D 48, 2728 (1993); R. Omnes The Interpretation of Quantum Mechanics Princeton Un. Press (1994), Chap. 7

[4] G.C. Ghirardi, A. Rimini, T. Weber Phys. Rev. D 34, 470, (1986)

[5] P. Pearl, Phys. Rev. A 39, 2277 (1989); G. C. Ghirardi, P. Pearle, A. Rimini Phys. Rev. A 42, 78 (1990)

[6] M. Cini, M. Serva Foundations of Physics Letters, 3, 129, (1990).

[7] L.D. Landau and E.M. Lifshitz Statistical Physics Pergamon Press, London (1958) p. 14-15 ; E. Wigner in Foundations of Quantum Mechanics, edited by B. d'Espagnat, Academic Press , New York (1971) p. 1.

[8] M. Tegmark, Foundations of Physics Letters, 6, 571, (1993) 
[9] A. deShalit and H. Feshbach Theoretical Nuclear Physics John Wiley and Sons, New York (1969), Vol.I p. 191-194

[10] P. Poll, N.W. Ashcroft, Phys. Rev. A 32, 1722 (1985)

[11] J.A. Barker Rare Gas Solids Vol. 1, M.L. Klein, J.A. Venables eds., Academic Press, London 1976 p. 212

[12] B. Carazza, P. Quarati, N. C. 106 A, 1353 (1993)

[13] J.M. Levi-Leblond, Rivista del N.C., 4, 99 (1974)

[14] J. M. Levi-Leblond, J. Math. Phys., 10, 806 (1969) 